\title{
ON INTERSECTIONS AND UNIONS OF RADICAL CLASSES
}

\author{
YU-LEE LEE and R. E. PROPES ${ }^{1}$ \\ (Received 20 August 1969; revised 12 November 1969 \\ Communicated by B. Mond
}

\section{Introduction}

Let $\mathscr{A}$ be a class of rings, and let $L(\mathscr{A})$ denote the lower radical class determined by $\mathscr{A}$. In [3] Yu-Lee Lee showed that $L(\mathscr{A})$ may be constructed in the following manner: Let $H(\mathscr{A})$ be the class of all homomorphic images of rings in $\mathscr{A}$. For each ring $R$, let $D_{1}(R)$ be the set of all ideals of $R$, and by induction define $D_{n+1}(R)$ to be the family of all rings which are ideals of some ring in $D_{n}(R)$ and set $D(R)=\cup\left\{D_{n}(R): n=1,2,3, \cdots\right\}$ which is commonly known as the hereditary closure of $R$. A ring $R$ is called an $L(\mathscr{A})$ - ring if $D(R / I)$ contains a nonzero ring which is isomorphic to a ring in $H(\mathscr{A})$ for each ideal $I$ of $R$ and $I \neq R$ i.e., if each non-zero homomorphic image of $R$ contains an accessible subring isomorphic to a ring in $H(\mathscr{A})$. In [4] Yu-Lee Lee proved that any class $\mathscr{A}$ of rings determines an upper radical property $\subseteq(\mathscr{A})$.

The following theorem was conjectured by Yu-Lee Lee: Let $\mathscr{A}_{i}$ be a homomorphically closed and hereditary class of rings $(i=1,2)$. Then $L\left(\mathscr{A}_{1} \cap \mathscr{A}_{2}\right)=$ $L\left(\mathscr{A}_{i}\right) \cap L\left(\mathscr{A}_{2}\right)$. The purpose of this paper is to prove this theorem and, in addition, to prove an "intersection theorem" for upper radicals. In this paper we shall use the following notation: $I \leqq R$ signifies that $I$ is an ideal of the ring $R$.

We shall use the following theorem which is due to A. E. Hoffman and W. G. Leavitt [2]. Theorem. If $\mathscr{A}$ is a hereditary class, then $L(\mathscr{A})$ is hereditary.

Since we shall be concerned with the intersections of radical classes, we shall often employ (without specifically noting it) the following useful proposition. We mention that $T$. L. Jenkins in [1] proved an analogous proposition for hereditary radicals.

Proposition. Let $P_{1}$ and $P_{2}$ be radical classes in some universal class $\mathscr{W}$ of rings, and define $T(R)=P_{1}(R) \cap P_{2}(R)$, and set $T=\{R \in \mathscr{W}: T(R)=R\}$. Then $T=P_{1} \cap P_{2}$.

Proof. $\quad R \in T$ iff $R=T(R)=P_{1}(R) \cap P_{2}(R)$

$$
\begin{aligned}
& \text { iff } R=P_{1}(R)=P_{2}(R) \\
& \text { iff } R \in P_{1} \cap P_{2} .
\end{aligned}
$$

1 This research was supported partially by Kansas State University Research Grant No. F 440 . 
2.

THEOREM 1. Let $\mathscr{A}_{i}$ be a homomorphically closed and hereditary class of rings $(i=1,2)$. Then $L\left(\mathscr{A}_{1} \cap \mathscr{A}_{2}\right)=L\left(\mathscr{A}_{1}\right) \cap L\left(\mathscr{A}_{2}\right)$.

ProOf. Since $L\left(\mathscr{A}_{1} \cap \mathscr{A}_{2}\right) \subseteq L\left(\mathscr{A}_{i}\right)$ for $i=1,2$, we have $L\left(\mathscr{A}_{1} \cap \mathscr{A}_{2}\right)$ $\subseteq L\left(\mathscr{A}_{1}\right) \cap L\left(\mathscr{A}_{2}\right)$. Thus let $R \in L\left(\mathscr{A}_{1}\right) \cap L\left(\mathscr{A}_{2}\right)$ and let $I$ be a proper ideal of $R$. Now $R \in L\left(\mathscr{A}_{1}\right)$ implies $D(R / I) \cap \mathscr{A}_{1} \neq 0$, hence let $A \in D(R / I) \cap \mathscr{A}_{1}$. Since $L\left(\mathscr{A}_{1}\right) \cap L\left(\mathscr{A}_{2}\right)$ is hereditary [2] and since $R / I \in L\left(\mathscr{A}_{1}\right) \cap L\left(\mathscr{A}_{2}\right)$, we have $D(R / I) \subseteq L\left(\mathscr{A}_{1}\right) \cap L\left(\mathscr{A}_{2}\right)$; and so $A \in L\left(\mathscr{A}_{1}\right) \cap L\left(\mathscr{A}_{2}\right)$, viz., $A \in L\left(\mathscr{A}_{2}\right)$. But $A \in L\left(\mathscr{A}_{2}\right)$ implies $D(A) \cap \mathscr{A}_{2} \neq 0$. Thus let $0 \neq B \in D(A) \cap \mathscr{A}_{2}$. Now $\mathscr{A}_{1}$ is hereditary, and $A \in \mathscr{A}_{1}$, so that $D(A) \subseteq \mathscr{A}_{1}$. Hence $B \in \mathscr{A}_{1} \cap \mathscr{A}_{2}$. But $D(A)$ $\subseteq D(R / I)$ so that $B \in D(R / I) \cap\left(\mathscr{A}_{1} \cap \mathscr{A}_{2}\right)$. Therefore $D(R / I) \cap\left(\mathscr{A}_{1} \cap \mathscr{A}_{2}\right)$ $\neq 0$. Thus $R \in L\left(\mathscr{A}_{1}\right) \cap L\left(\mathscr{A}_{2}\right)$ implies $R \in L\left(\mathscr{A}_{1} \cap \mathscr{A}_{2}\right)$. This completes the proof of Theorem 1 .

Note. By an inductive argument $L\left(\bigcap_{i=1}^{n} \mathscr{A}_{i}\right)=\bigcap_{i=1}^{n} L\left(\mathscr{A}_{i}\right)$, where $\mathscr{A}_{i}$ is a class of rings which is both homomorphically closed and hereditary for $i=$ $1,2, \cdots, n$. Next we provide an example for which $L(\mathscr{A} \cap \mathscr{B})$ is a proper subset of $L(\mathscr{A}) \cap L(\mathscr{B})$.

EXAMPLE. Let $Z$ denote the ring of integers, and let (4) denote the principal ideal of $Z$ generated by the integer 4 . Let $Z /(4)$ denote the ordinary quotient ring, and let $R=\{0+(4), 2+(4)\}$. Let $\mathscr{A}=H(\{(Z /(4)) / R, R\})$ and $\mathscr{B}=H(\{Z /(4)\})$ denote the homomorphic closures of the classes $\{(Z /(4)) / R, R\}$ and $\{Z /(4)\}$ respectively. It is easy to see that the only proper ideal of $Z /(4)$ is $R$ and the ring $Z /(4)$ cannot be mapped homomorphically onto its ideal $R$. We also note that the only subrings of $Z /(4)$ are $Z /(4), R, 0 \cdots$ none of which is a field (hence none is isomorphic with $(Z /(4)) / R)$.

Now $\mathscr{A} \cap \mathscr{B}=H(\{(Z /(4)) / R\})$, and $(Z /(4)) / R$ is simple; therefore each ring in $\mathscr{A} \cap \mathscr{B}$ is either 0 or else isomorphic with $(Z /(4)) / R$. Since $Z /(4)$ has no subring isomorphic to the field $(Z /(4)) / R$, then $Z /(4) \notin L(\mathscr{A} \cap \mathscr{B})$, in fact, $L(\mathscr{A} \cap \mathscr{B})$ $(Z /(4))=0$. Certainly $Z /(4) \in L(\mathscr{B})$, and also $Z /(4) \in L(\mathscr{A})$, because each nonzero homomorphic image of $Z /(4)[(Z /(4)) / R, Z /(4)]$ contains a non-zero subring in $\mathscr{A}$. Thus $Z /(4) \in L(\mathscr{A}) \cap L(\mathscr{B})$ and hence $L(\mathscr{A} \cap \mathscr{B})$ is a proper subset of $L\left(\mathscr{A}_{1}\right) \cap L\left(\mathscr{A}_{2}\right)$.

We note that $\mathscr{A}$ is hereditary, because both $(Z /(4)) / R$ and $R$ are simple rings. However, $\mathscr{B}$ is not hereditary, because $R \leqq Z /(4) \in \mathscr{B}$, but $R \notin \mathscr{B}$.

For a class $\mathscr{M}$ of rings, let $\subseteq(\mathscr{M})$ denote the upper radical class determined by the class $\mathscr{M}$. The following theorem is similar to 2.3 .3 of $[1$, p. 28].

THEOREM 2. Let $\mathscr{A}$ and $\mathscr{B}$ be classes of rings. Then $S(\mathscr{A} \cup \mathscr{B})=$ $\subseteq(\mathscr{A}) \cap \subseteq(\mathscr{B})$.

Proof. First, since each ring in $\mathscr{A}$ is in $\mathscr{A} \cup \mathscr{B}$, then each ring in $\mathscr{A}$ is 


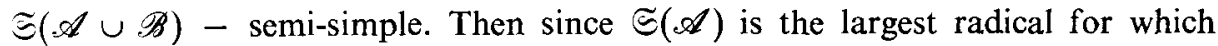
every ring in $\mathscr{A}$ is semi-simple, we must have $S(\mathscr{A} \cup \mathscr{B}) \subseteq \subseteq(\mathscr{A}$.) Similarly

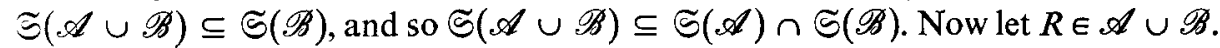
If $R \in \mathscr{A}$, then $(\subseteq(\mathscr{A}))(R)=0$ and so $(\subseteq(\mathscr{A}) \cap \subseteq(\mathscr{B}))(R)=0$. Similarly, for $R \in \mathscr{B},(\mathscr{S}(\mathscr{A}) \cap \subseteq(\mathscr{B}))(R)=0$. Thus every ring in $\mathscr{A} \cup \mathscr{B}$ is $\subseteq(\mathscr{A}) \cap \subseteq(\mathscr{B})$ semi-simple. Since $\subseteq(\mathscr{A} \cup \mathscr{B})$ is the largest radical for which every ring in $\mathscr{A} \cup \mathscr{B}$ is semi-simple, we must have $\subseteq(\mathscr{A}) \cap \subseteq(\mathscr{B}) \subseteq \subseteq(\mathscr{A} \cup \mathscr{B})$. Hence $\overleftarrow{S}(\mathscr{A} \cup \mathscr{B})=\subseteq(\mathscr{A}) \cap \mathfrak{S}(\mathscr{B})$.

\section{References}

[1] T. L. Jenkins, The theory of radicals and radical rings (Ph.D. thesis, University of Nebraska, 1966).

[2] A. E. Hoffman \& W. G. Leavitt, 'Properties inherited by the lower radical' (to appear).

[3] Yu-Lee Lee, 'On the construction of lower radical properties of rings', Pacific Journal of Math. (to appear).

[4] Yu-Lee Lee, 'On the construction of upper radical properties', Proc. Amer. Math. Soc. 19, No. 5, (1968).

Kansas State University

and

New York State University College

at Potsdam 